\title{
Can the American Thyroid Association Risk of Recurrence Predict Radioiodine Refractory Disease in Differentiated Thyroid Cancer?
}

\author{
Aamna Hassan ${ }^{a}$ Saima Riaz ${ }^{a}$ Humayun Bashir ${ }^{a} \quad$ M. Khalid Nawaz ${ }^{a}$ \\ Raza Hussain ${ }^{b}$
}

Departments of a Nuclear Medicine and ${ }^{\mathrm{b}}$ Surgical Oncology, Shaukat Khanum Memorial Cancer Hospital and

Research Centre, Lahore, Pakistan

\section{Key Words}

Radioiodine refractory disease $\cdot$ Differentiated thyroid cancer - American Thyroid Association risk classification .

TNM in iodine resistance $\cdot{ }^{18} \mathrm{~F}$-fludeoxyglucose PET/CT scan

\begin{abstract}
Objective: The aim of this study was to compare the TNM staging system and the American Thyroid Association (ATA) recurrence risk classification in predicting radioiodine refractory disease (RRD) in differentiated thyroid cancer (DTC) and to analyze the correlation of stimulated thyroglobulin $(\mathrm{Tg})$ levels and rate of $\mathrm{Tg}$ elevation with the standardized uptake value on ${ }^{18} \mathrm{~F}$-fludeoxyglucose (FDG) PET/CT scan. Methods: RRD was indicated through the retrospective analysis of consecutive ${ }^{18} \mathrm{~F}$-FDG PET/CT scans in DTC with stimulated Tg $>10 \mathrm{ng} / \mathrm{ml}$ and negative ${ }^{131}$ I Nal whole-body scans (WBS). Tg elevation velocity was compared to the likelihood of a positive scan. The ATA recurrence risk and TNM staging system were compared to see which of them better predicted the subsequent development of RRD. Results: Fifty-eight of 636 subjects developed RRD: 52 papillary and 6 follicular thyroid cancer. The median time between diagnosis and a negative WBS was 24 months (range 12-240). RRD developed in 11 low-risk, 32 intermediate-risk and 15 high-risk patients. A
\end{abstract}

better response to therapy was seen in the low-risk versus the intermediate- and high-risk groups. ${ }^{18} \mathrm{~F}$-FDG PET/CT scans had a diagnostic accuracy of $94.8 \%$, sensitivity of $97.7 \%$, specificity of $85.7 \%$, positive predictive value of $95.6 \%$ and negative predictive value of $92 \%$. There was no correlation between the $\mathrm{Tg}$ level or rate of rise and a positive scan. Overall, PET-CT upstaged 18 (31\%) cases, leading to a change in management in 20 (35\%) cases. Conclusion: The TNM and ATA staging systems show no significant difference in predicting the development of RRD. RRD is less likely in stage I, II and low-risk patients. There is no correlation between the level or rate of Tg rise and a positive ${ }^{18} \mathrm{~F}$-FDG PET/CT scan.

(c) 2016 European Thyroid Association Published by S. Karger AG, Basel

\section{Introduction}

Differentiated thyroid cancer (DTC) is typically indolent if treated early and appropriately. A small subset of more aggressive disease progresses to radioiodine resistance. Based on the American Thyroid Association (ATA) risk of recurrence classification at baseline [1] and response to initial therapy 6-24 months after radioactive iodine (RAI) ablation [2], the more aggressive subset of

\begin{tabular}{|c|c|}
\hline KARGER & $\begin{array}{l}\text { (๑) } 2016 \text { European Thyroid Association } \\
\text { Published by S. Karger AG, Basel }\end{array}$ \\
\hline $\begin{array}{l}\text { E-Mail karger@karger.com } \\
\text { www.karger.com/etj }\end{array}$ & $2235-0640 / 16 / 0054-0261 \$ 39.50 / 0$ \\
\hline
\end{tabular}

Dr. Aamna Hassan, MBBS, MD, CCD

Department of Nuclear Medicine, Shaukat Khanum Memorial Cancer Hospital and Research Centre, 7-A, Block R-3

Johar Town, Lahore 54000 (Pakistan)

E-Mail aamnah@ skm.org.pk 
patients should theoretically be identified in whom the chance of developing radioiodine refractory disease (RRD) would potentially be higher. There has so far been no study defining the role of ATA risk stratification or TNM classification in predicting which patients will progress to develop iodine resistance/dedifferentiation with biochemical evidence of disease presence.

Although the ${ }^{18}$ F-fludeoxyglucose (FDG) PET/CT scan has a well-established role in detecting disease in RRD [1], the relation of the rate of thyroglobulin (Tg) rise or stimulated Tg level with the likelihood of disease detection has not been established. The purpose of our study was to (1) stratify the RRD patients based on ATA risk and TNM staging systems to see which of the two, if either, can predict the subsequent development of iodine resistance; (2) evaluate the association of $\mathrm{Tg}$ rise velocity to ${ }^{18} \mathrm{~F}-\mathrm{FDG}$ PET/CT scan positivity and study the role of the ${ }^{18} \mathrm{~F}$-FDG PET/CT scan in the further management of these patients, and (3) study the subsequent behavior of RRD after directed treatment.

\section{Methods}

\section{Setting and Data Collection}

The study was performed at the department of Nuclear Medicine at Shuakat Khanum Memorial Cancer Hospital and Research Centre, a cancer specialist center in Lahore, Pakistan. A retrospective analysis was conducted of all ${ }^{18} \mathrm{~F}-\mathrm{FDG}$ PET/CT scans performed over 5 years in DTC patients with stimulated Tg levels $>10$ $\mathrm{ng} / \mathrm{ml}$ or positive anti-Tg antibody (TgAb) and a negative ${ }^{131} \mathrm{I} \mathrm{NaI}$ whole-body scan (WBS). Patients were classified based on their TNM stage and ATA risk categorization.

\section{Clinical Management}

At baseline all patients underwent total thyroidectomy and RAI remnant ablation. Patients were followed up regularly every 6-12 months, as per the standard protocol. We classified our patients as low, intermediate and high risk according to the 2010 ATA guidelines for recurrence risk classification.

ELISA testing of Tg levels was done using a chemiluminescence immunoassay analyzer. A Tg value $<0.2 \mathrm{ng} / \mathrm{ml}$ was considered normal in postthyroidectomy patients. In $\mathrm{TgAb}$ cases, a level of $>20 \mathrm{IU} / \mathrm{ml}$ was considered elevated. In such cases, the Tg value was regarded as invalid and the disease status was evaluated by following the TgAb levels.

The ${ }^{18} \mathrm{~F}$-FDG PET/CT scans were performed on a dedicated Phillips Gemini TOF scanner. After a 4- to 6-hour fast, $300 \mathrm{MBq}$ of ${ }^{18}$ F-FDG was injected intravenously followed by a 60 -min uptake period. Three-minute images were acquired from the base of the skull to mid-thigh for each of 8-9 bed positions. A contrastenhanced CT scan was acquired with a voltage of $70-140 \mathrm{kVp}$ and tube current of $80 \mathrm{~mA}$ for attenuation correction and lesion localization. Iodinated contrast was not administered. Each scan was reported by a board-certified nuclear medicine physician and a radiologist. Regions of interest were drawn on attenuation-corrected images to calculate standardized uptake values (SUV) normalized to body weight. Maximum SUV (SUVmax) was calculated after rescaling the overall activity concentration $\left(\mathrm{Bq} \mathrm{ml}^{-1}\right)$. The uptake of all abnormal sites was recorded. The hepatic SUVmax was used as the reference value.

Based on ${ }^{18} \mathrm{~F}-\mathrm{FDG}$ PET/CT scan findings, if the hypermetabolic lesion was accessible, surgical resection was the preferred option. If surgery was not possible, external beam radiation therapy was performed in symptomatic patients. Where neither was a viable option an empirical RAI therapy was administered. In instances where there was an overlap of two or three of these modalities, we evaluated the response to optimal treatment and classified it as biochemically complete, biochemically incomplete, structural and biochemical progression, or death.

\section{Data Analysis}

Patient demographics and clinical characteristics were assessed using standard descriptive statistics applicable on quantitative and qualitative data. Nonparametric correlation of Tg levels, ATA risk stratification and TNM versus ${ }^{18} \mathrm{~F}$-FDG PET/CT outcome were assessed by applying Spearman's correlation.

The Tg velocity was calculated taking into account the stimulated Tg levels, approximately 12 months apart, using the formula: pre- ${ }^{18}$ F-FDG PET/CT Tg - Tg on last follow-up/pre- ${ }^{18}$ F-FDG PET/CT Tg $\times 100$. Spearman's correlation between the Tg velocity and time duration was assessed. $p$ values $<0.05$ were considered statistically significant. The study was approved by the institutional review board.

\section{Results}

Out of a total of 954 patients with DTC registered at our institute over a 19-year period, 636 patients (538 papillary cancer, 98 follicular cancer) were analyzed. In total, 58 (9\%) developed RRD during follow-up. This included 33 (57\%) females and 25 (43\%) males; 52 (90\%) had papillary cancer and $6(10 \%)$ had follicular cancer.

The median time between DTC diagnosis and a negative ${ }^{131} \mathrm{I}$ WBS was 24 months (range $12-240$ ). Pre- ${ }^{18} \mathrm{~F}$ FDG PET/CT stimulated Tg levels ranged from 10 to $17,355 \mathrm{ng} / \mathrm{ml}$. Five patients underwent ${ }^{18} \mathrm{~F}$-FDG PET/CT scan due to rising $\mathrm{TgAb}$ levels.

Out of $58{ }^{18} \mathrm{~F}-\mathrm{FDG}$ PET/CT scans, 43 (74\%) were positive and 37 showed FDG avid disease, while 6 had subcentimeter non-FDG avid pulmonary nodules which were below the scan resolution. Two with nodal uptake were found to be reactive nodes on histology, representing a false positive finding. Twelve $(20.6 \%)$ showed no uptake or morphologic abnormality. The false negative rate was $1.7 \%$. One patient had no evidence of FDG avid disease; however, neck dissection confirmed nodal metastases. 
Table 1. Stratification of data based on ATA low-, intermediate- and high-risk categories

\begin{tabular}{lccc}
\hline & $\begin{array}{l}\text { Low risk } \\
(\mathrm{n}=11)\end{array}$ & $\begin{array}{l}\text { Intermediate } \\
\text { risk }(\mathrm{n}=32)\end{array}$ & $\begin{array}{l}\text { High risk } \\
(\mathrm{n}=15)\end{array}$ \\
\hline Female/male, \% & $50 / 50$ & $54.5 / 45.5$ & $60 / 40$ \\
Mean stimulated Tg, ng/ml & $228(10-1,144)$ & $764(21-17,355)$ & $570(58-1,623)$ \\
Mean time to negative WBS, months & $42(12-84)$ & $30.5(12-240)$ & $36(12-72)$ \\
Mean SUVmax & $5.7(2.4-14.5)$ & $5.3(2.6-16)$ & $8.7(4.4-22.6)$ \\
\hline
\end{tabular}

The diagnostic accuracy of ${ }^{18} \mathrm{~F}-\mathrm{FDG}$ PET/CT scan was $94.8 \%$, sensitivity $97.7 \%$, specificity $85.7 \%$, positive predictive value $95.6 \%$, and negative predictive value $92 \%$. The ${ }^{18} \mathrm{~F}-\mathrm{FDG}$ PET/CT scans led to a change in management in $34 \%$ of patients.

The SUV of positive sites ranged from 2.6 to 22.6. There was no correlation between the Tg level and scan negativity $(\mathrm{r}=0.093, \mathrm{p}=0.51)$, and no association between SUV and Tg levels $(r=0.13, p=0.33)$ in the positive scans. An overview of the ${ }^{18} \mathrm{~F}-\mathrm{FDG}$ PET/CT results in relation to Tg levels is provided in figure 1 . Tg level was not found to be useful in determining PET/CT positivity. The ROC curve showed an AUC of 0.568 ( $\mathrm{p}=0.508,95 \%$ CI $0.365-0.771)$.

Tg velocities were calculated to evaluate the rate of change. The data were analyzed based on the ATA risk categories. There was no statistically significant correlation between $\mathrm{Tg}$ velocity and the likelihood of a positive ${ }^{18} \mathrm{~F}-\mathrm{FDG}$ PET/CT scan in the low- $(\mathrm{r}=0.087, \mathrm{p}=0.81)$, intermediate- $(\mathrm{r}=0.395, \mathrm{p}=0.069)$ or high-risk groups $(\mathrm{r}=0.318, \mathrm{p}=0.290)$.

\section{ATA Risk Stratification}

On the basis of the ATA risk classification, $4.5 \%$ of 243 low-risk patients, $11.6 \%$ of 238 intermediate-risk patients, and $12.8 \%$ of 117 high-risk patients developed RRD (table 1).

\section{Low-Risk Group}

The low-risk group consisted of 11 patients (18.9\%). The ${ }^{18} \mathrm{~F}$-FDG PET/CT scan was positive in $73 \%$ of the cases. Hypermetabolic disease was identified in 7 patients (63.6\%), of whom 1 had false positive uptake in a neck node found to be reactive on histology. Nonavid structural disease was identified in $1(9 \%)$ patient, while the ${ }^{18}$ F-FDG PET/CT was unremarkable in 3 (27\%) cases. Two out of 11 had elevated anti-Tg (TgAb) levels.

The PET-CT scan upstaged disease in 3 (27\%) patients. Most patients with a positive ${ }^{18} \mathrm{~F}-\mathrm{FDG} \mathrm{PET} / \mathrm{CT}$ scan were

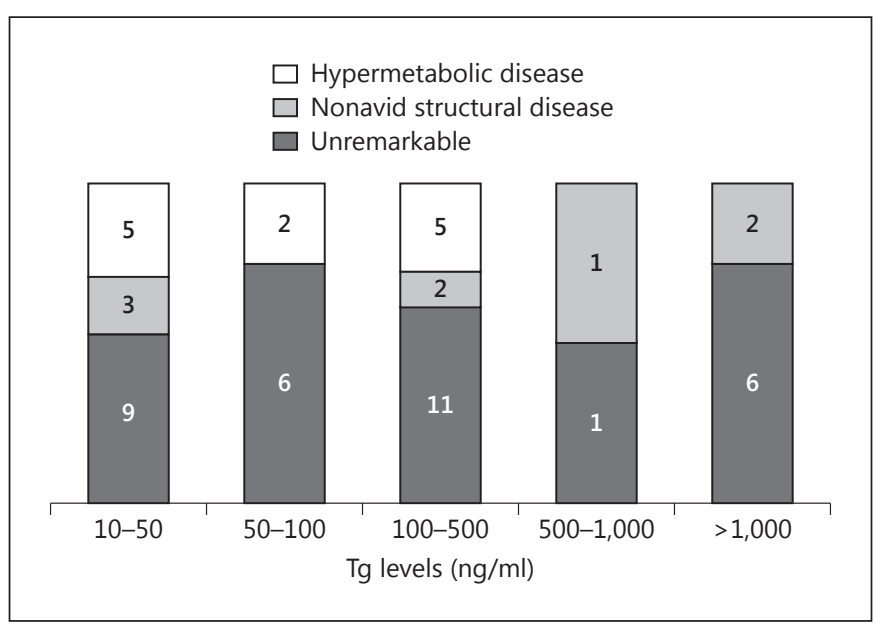

Fig. 1. ${ }^{18} \mathrm{~F}$-FDG PET/CT outcome in relation to Tg levels.

given empirical RAI therapy, and surgical resection of avid disease was performed in $3(27 \%)$ patients. The details of the subsequent follow-up of these low-risk patients are presented in figure 2 . In all 3 patients with a negative ${ }^{18}$ F-FDG PET/CT scan, stimulated Tg levels normalized after an empirical dose of RAI.

\section{Intermediate-Risk Group}

Thirty-two (55.2\%) patients had an intermediate risk of recurrence at baseline. Out of these, the ${ }^{18} \mathrm{~F}-\mathrm{FDG}$ PET/ CT scan was positive in $78 \%$ : hypermetabolic disease was identified in 23 (72\%), nonavid pulmonary nodules were identified in $2(6.2 \%)$, and $7(22 \%)$ were negative. Of these, 2 had elevated TgAb levels.

The PET/CT scan upstaged the disease in 10 (31\%) patients. Of 25 patients with a positive ${ }^{18} \mathrm{~F}$-FDG PET/CT scan, $10(40 \%)$ underwent surgical resection or radiotherapy where applicable, and 23 were given empirical RAI therapy, alone or in combination with surgery or radiation. Details of the subsequent follow-up of these patients 
Fig. 2. ATA risk-based groups with post${ }^{18} \mathrm{~F}-\mathrm{FDG}$ PET/CT clinical outcome.

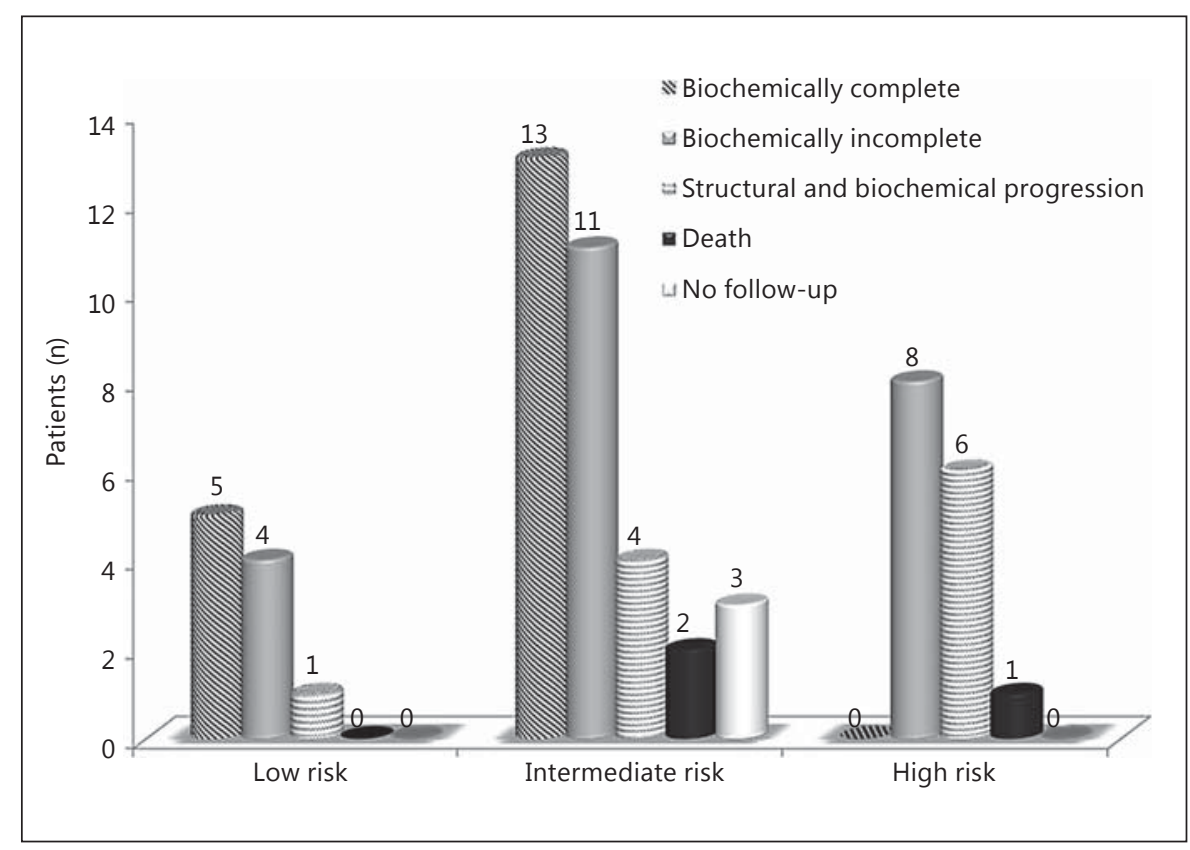

Table 2. ATA risk groups and ${ }^{18} \mathrm{~F}-\mathrm{FDG}$ PET/CT outcome

\begin{tabular}{lclll}
\hline ATA risk & ${ }^{18}$ F-FDG PET-CT outcome & Total \\
\cline { 2 - 4 } & $\begin{array}{l}\text { hypermetabolic } \\
\text { disease }\end{array}$ & unremarkable & $\begin{array}{l}\text { nonavid } \\
\text { structural } \\
\text { disease }\end{array}$ & \\
\hline Low & $7(63.6)$ & $3(27.3)$ & $1(9)$ & 11 \\
Intermediate & $23(72)$ & $7(21.8)$ & $2(6.2)$ & 32 \\
High & $9(60)$ & $2(13.3)$ & $4(26.7)$ & 15 \\
\hline
\end{tabular}

Data are presented as n (\%).

with an intermediate risk of recurrence are displayed in figure 2.

\section{High-Risk Group}

Fifteen (25.9\%) patients were classified as high risk at baseline. Out of these, the ${ }^{18} \mathrm{~F}-\mathrm{FDG}$ PET/CT scan was positive in $13(87 \%)$ cases, with 9 showing hypermetabolic structural disease and 4 displaying nonavid pulmonary nodules. The ${ }^{18} \mathrm{~F}-\mathrm{FDG}$ PET/CT scan was negative in $2(13 \%)$ cases. Both showed progression on follow-up and 1 had elevated TgAb levels.

The PET-CT scan upstaged disease in 5 (33.3\%) patients. Surgery and radiation therapy was given in $7(47 \%)$ cases and $9(60 \%)$ received empirical RAI therapy. Details of the subsequent follow-up of these patients with a high risk of recurrence are presented in figure 2. Out of $46 \mathrm{pa}-$ tients who were given an empirical dose, radioiodine avid disease was demonstrated in $14 \%$ on posttherapy scans.

\section{Elevated TgAb Level}

In 5 subjects, the ${ }^{18} \mathrm{~F}-\mathrm{FDG} \mathrm{PET} / \mathrm{CT}$ scan was acquired for elevated $\mathrm{TgAb}$ with a negative RAI diagnostic WBS (low risk, $\mathrm{n}=2$, intermediate risk, $\mathrm{n}=1$, and high risk, $\mathrm{n}=2$; TgAb range $341-3,000$ ). All had hypermetabolic disease on their ${ }^{18}$ F-FDG PET/CT scan. Follow-up after appropriate therapy resulted in a biochemical incomplete response in 4 with further elevation in TgAb levels, while in 1 case the levels declined. There was no statistically significant correlation between the ATA risk categories and the chance of developing $\mathrm{RRD}$ ( $\mathrm{p}=0.117$; table 2; fig. 3 ).

\section{TNM Stage}

On the basis of TNM staging, 7.3\% of 436 stage I patients, $8.6 \%$ of 58 stage II patients, $15 \%$ of 46 stage III patients and $14 \%$ of 96 stage IV patients developed RRD. There was no statistically significant correlation between the TNM stage and development of RRD ( $p=0.221$; fig. 4).

\section{ATA Risk Stratification versus TNM Stage}

On bivariate analysis the ATA classification (hazard ratio $1.31,95 \%$ CI $0.83-2.06, \mathrm{p}=0.24$ ) was a better pre- 


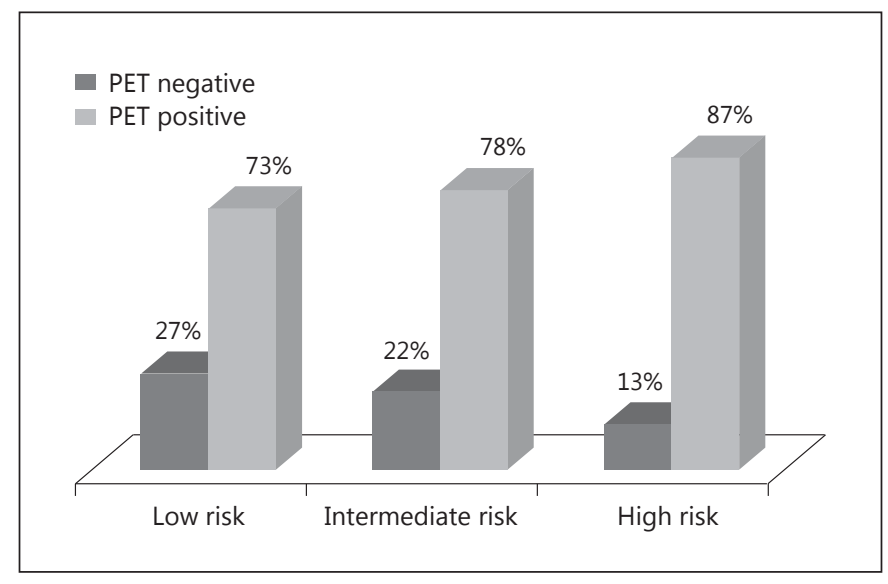

Fig. 3. ${ }^{18}$ F-FDG PET-CT and RRD in the ATA risk groups.

dictive staging system than the TNM classification (hazard ratio $0.903,95 \%$ CI $0.70-1.16, p=0.41)$. However, this difference in predictive value was not statistically significant. Both TNM staging and ATA risk assessment did not predict the chance of a positive PET/CT scan once RRD had developed (fig. 3, 4).

\section{Discussion}

DTC develops radioiodine resistance in approximately $2-11 \%$ of cases, and physicians are urged to enter such patients into clinical trials where possible [3]. Following thyroidectomy, patients are treated with RAI and monitored with WBS using RAI, serum tumor markers, Tg and $\mathrm{TgAb}$, and cervical ultrasonography at intervals of 6-12 months with individualized care plans. Radioiodine resistance in DTC represents an aggressive subset of patients, and once iodine resistance develops the disease becomes more challenging.

Details of the overall survival of our DTC population have already been published $[4,5]$. In the present study, although ${ }^{18} \mathrm{~F}-\mathrm{FDG} \mathrm{PET} / \mathrm{CT}$ played an instrumental role in detecting sites of disease in RRD, there was no clear-cut prediction of the development of RRD using the TNM staging or ATA risk assessment systems. An increase in FDG uptake is typically seen in more aggressive, highgrade thyroid cancer [6]. In our study there was no association between the chance of a positive FDG scan and the TNM stage or ATA risk category.

At our institution, the iodine-resistant population was treated aggressively with surgical resection and, if indi-

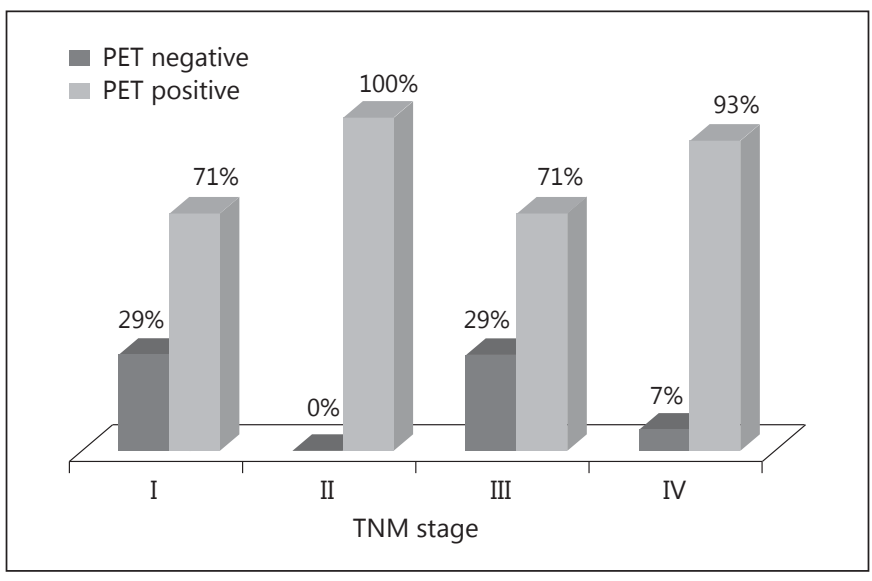

Fig. 4. ${ }^{18}$ F-FDG PET-CT and RRD according to TNM stage.

cated, radiation therapy based on ${ }^{18} \mathrm{~F}-\mathrm{FDG} \mathrm{PET} / \mathrm{CT}$ findings. RAI therapy was given to several patients in the earlier years when conflicting data were available as to the efficacy of empirical RAI therapy. Several studies have shown that when a diagnostic WBS is negative, empirical RAI therapy is not useful diagnostically or therapeutically in patients with negative ${ }^{18} \mathrm{~F}-\mathrm{FDG}$ PET/CT findings [7]. However, according to $\mathrm{Ma}$ et al. [8] Tg levels decreased after RAI therapy in $63 \%$ of patients, while in $62 \%$ there was uptake on the posttherapy scan. Pineda et al. [9] showed a decrease in follow-up Tg levels in $81 \%$ of patients undergoing first-line therapy, $90 \%$ undergoing second-line therapy, and in $100 \%$ of patient undergoing third-line therapy.

In our study, 12 patients had no metabolic or morphologic evidence of disease on their ${ }^{18} \mathrm{~F}$-FDG PET/CT scan although their Tg levels were elevated. According to Vural et al. [10] a negative ${ }^{18} \mathrm{~F}-\mathrm{FDG}$ PET/CT is a good prognostic indicator of prolonged symptom-free survival and, therefore, empirical therapy may not be justified. In some patients $\mathrm{Tg}$ follows a fluctuant course, and biochemically incomplete response to therapy may with time convert to no evidence of disease with a normalization of Tg levels.

The sensitivity of ${ }^{18} \mathrm{~F}$-FDG PET/CT in our population was very high, which is in line with other studies. Ong et al. [11] and Nahas et al. [12] reported a sensitivity of $88 \%$, while the latter group also reported a specificity of $100 \%$ and positive predictive value of $100 \%$. Petrich et al. [13] reported the sensitivity of ${ }^{18} \mathrm{~F}-\mathrm{FDG} \mathrm{PET} / \mathrm{CT}$ to be $53 \%$ during thyroid-stimulating hormone (TSH) suppression and $87 \%$ following recombinant human (rh) TSH stimulation. 
We did not find any relation between the actual level of $\mathrm{Tg}$ and the likelihood of a positive ${ }^{18} \mathrm{~F}-\mathrm{FDG}$ PET/CT scan. Similarly, there was no correlation with the rate of rise of $\mathrm{Tg}$ and the probability of a positive scan. This is contrary to the findings of Schluter et al. [14], who reported a positive scan in $11 \%$ of patients with Tg levels of $10 \mathrm{ng} / \mathrm{ml}$ or less, increasing to $50 \%$ when $\mathrm{Tg}$ levels were between 10 and $20 \mathrm{ng} / \mathrm{ml}$, and rising to $93 \%$ at Tg levels above $100 \mathrm{ng} / \mathrm{ml}$. In a study of 40 patients with negative ${ }^{131}$ I scans and elevated TgAb levels, Asa et al. [15] reported a positive ${ }^{18} \mathrm{~F}$-FDG PET/CT in $50 \%$ and concluded that ${ }^{18}$ F-FDG PET/CT can be used effectively in the detection of recurrence/metastasis during follow-up.

The limitations of our study include the fact that the data were retrospectively gathered. However, it is difficult to gather prospective data for the evaluation of thyroid cancer since the disease expectancy is long and decades of follow-up are not conveniently recorded prospectively.

Our study spanned 2 decades of data, and since the understanding of disease behavior has evolved over this time frame there was variation in treatment choices. In the last decade microcarcinoma has been defined as $<1$ $\mathrm{cm}$ in size, while prior to that no difference in treatment was made based on size.

Furthermore, empirical RAI therapy was initially given to all patients with elevated $\mathrm{Tg}$ levels, even when no iodine avid disease was seen on the pretherapy scan. Our center did not have a PET/CT scanner before 2009 and, therefore, at that time patients with RRD did not have an ${ }^{18}$ F-FDG PET/CT scan and instead underwent RAI therapy followed by a posttherapy scan.

Others report that, due to the frequent normalization of Tg levels in untreated patients, RAI therapy should be administered on a case by case basis [16]. Even if there is no evidence of radioiodine avid disease an empirical RAI therapy can be performed to identify the location of recurrent or metastatic disease [17-19]. An ${ }^{18}$ F-FDG PET/ $\mathrm{CT}$ scan may also serve a prognostic role considering that patients with high SUV and large-volume hypermetabolic disease have a higher 3-year mortality than those with nonavid disease on an ${ }^{18}$ F-FDG PET/CT scan $[20,21]$.

\section{Conclusion}

The TNM or ATA staging systems show no significant difference in predicting the development of RRD. RRD is less likely in stage I, II and low-risk patients. There is no correlation between the level or rate of Tg rise and a positive ${ }^{18} \mathrm{~F}-\mathrm{FDG}$ PET/CT scan.

\section{Disclosure Statement}

The authors have no conflicts of interest to report.

\section{References}

1 Tuttle RM, Tala H, Shah J, et al: Estimating risk of recurrence in differentiated thyroid cancer after total thyroidectomy and radioactive iodine remnant ablation: using response to therapy variables to modify the initial risk estimates predicted by the new American Thyroid Association staging system. Thyroid 2010;20:1341-1349.

2 Cooper DS, Doherty GM, Haugen BR, et al: Revised American Thyroid Association management guidelines for patients with thyroid nodules and differentiated thyroid cancer: The American Thyroid Association (ATA) Guidelines Taskforce on Thyroid Nodules and Differentiated Thyroid Cancer. Thyroid 2009;19:1167-1214.

3 Silberstein EB: The problem of the patient with thyroglobulin elevation but negative iodine scintigraphy: the TENIS syndrome. Semin Nucl Med 2011;41:113-120.
4 Hassan A, Khalid M, Riaz S, et al: Follicular thyroid carcinoma: disease response evaluation using American Thyroid Association risk assessment guidelines. Eur Thyroid J 2015;4: 260-265.

5 Hassan A, Razi M, Riaz S, Khalid M, Nawaz MK, Syed AA, Bashir H: Survival analysis of papillary thyroid carcinoma in relation to stage and recurrence risk: a 20-year experience in Pakistan. Clin Nucl Med 2016;41:606-613.

6 Grabellus F, Nagarajah J, Bockisch A, et al: Glucose transporter 1 expression, tumor proliferation, and iodine/glucose uptake in thyroid cancer with emphasis on poorly differentiated thyroid carcinoma. Clin Nucl Med 2012;37:121-127.

7 Kim WG, Ryu JS, Kim EY, et al: Empiric highdose 131-iodine therapy lacks efficacy for treated papillary thyroid cancer patients with detectable serum thyroglobulin, but negative cervical sonography and ${ }^{18} \mathrm{~F}$-fluorodeoxyglucose positron emission tomography scan. J Clin Endocrinol Metab 2010;95:1169-1173.
8 Ma C, Xie J, Kuang A: Is empiric 131I therapy justified for patients with positive thyroglobulin and negative 131I whole-body scanning results? J Nucl Med 2005;46:1164-1170.

$>9$ Pineda JD, Lee T, Ain K, et al: Iodine-131 therapy for thyroid cancer patients with elevated thyroglobulin and negative diagnostic scan. J Clin Endocrinol Metab 1995;80:14881492.

10 Vural GU, Akkas BE, Ercakmak N, et al: Prognostic significance of FDG PET/CT on the follow-up of patients of differentiated thyroid carcinoma with negative ${ }^{131}$ I whole-body scan and elevated thyroglobulin levels: correlation with clinical and histopathologic characteristics and long-term follow-up data. Clin Nucl Med 2012;37:953-959. 
11 Ong SC, Ng DC, Sundram FX: Initial experience in use of fluorine-18-fluorodeoxyglucose positron emission tomography/computed tomography in thyroid carcinoma patients with elevated serum thyroglobulin but negative iodine-131 whole body scans. Singapore Med J 2005;46:297-301.

12 Nahas Z, Goldenberg D, Fakhry C, et al: The role of positron emission tomography/computed tomography in the management of recurrent papillary thyroid carcinoma. Laryngoscope 2005;115:237-243.

13 Petrich T, Borner AR, Otto D, et al: Influence of rhTSH on $\left[{ }^{18} \mathrm{~F}\right]$ fluorodeoxyglucose uptake by differentiated thyroid carcinoma. Eur J Nucl Med Mol Imaging 2002;29:641-647.

14 Schluter B, Bohuslavizki KH, Beyer W, et al: Impact of FDG PET on patients with differentiated thyroid cancer who present with elevated thyroglobulin and negative ${ }^{131} \mathrm{I}$ scan. J Nucl Med 2001;42:71-76.
15 Asa S, Aksoy SY, Vatankulu B, et al: The role of FDG-PET/CT in differentiated thyroid cancer patients with negative iodine-131 whole-body scan and elevated anti-Tg level. Ann Nucl Med 2014;28:970-979.

16 Kamel N, Corapcioglu D, Sahin M, et al: I-131 therapy for thyroglobulin positive patients without anatomical evidence of persistent disease. J Endocrinol Invest 2004;27:949-953.

17 Leboulleux S, El Bez I, Borget I, et al: Postradioiodine treatment whole body scan in the era of fluorodeoxyglucose positron emission tomography for differentiated thyroid carcinoma with elevated serum thyroglobulin levels. Thyroid 2012;22:832-838.
8 Treglia G, Bertagna F, Piccardo A, et al: ${ }^{131} \mathrm{I}$ whole-body scan or ${ }^{18} \mathrm{FDG}$ PET/CT for patients with elevated thyroglobulin and negative ultrasound? Clin Transl Imaging 2013;1: 175-183.

19 Dong MJ, Liu ZF, Zhao K, et al: Value of $18 \mathrm{~F}$ FDG-PET/PET-CT in differentiated thyroid carcinoma with radioiodine negative wholebody scan: a meta-analysis. Nucl Med Commun 2013;30:639-650.

20 Deandreis D, Al Ghuzlan A, Leboulleux S, et al: Do histological, immunohistochemical, and metabolic (radioiodine and fluorodeoxyglucose uptakes) patterns of metastatic thyroid cancer correlate with patient outcome? Endocr Relat Cancer 2011;18:159-169.

-21 Robbins RJ, Wan Q, Grewal RK, et al: Realtime prognosis for metastatic thyroid carcinoma based on 2-[ $\left.{ }^{18} \mathrm{~F}\right]$ fluoro-2-deoxy-D-glucose-positron emission tomography scanning. J Clin Endocrinol Metab 2006;91: 498-505. 